\title{
Coartación Aórtica e Hipertensión Arterial
}

\author{
Dra. Soledad Tagle V. ${ }^{1}$, Dr. Carlos Sajeh A., ${ }^{2}$ Dr. Raúl Zilleruelo B. ${ }^{3}$
}

\section{Aortic Coarctation and Arterial Hypertension}

\begin{abstract}
The evolution of arterial pressure after surgical reparation in 40 patients with Coarctation of the Aorta, admitted to a children ${ }^{+}$Hospital (Luis Calvo Mackenna) between 1975 and 1981 is described. Ages at admission ranged from 5 moth to 15 years. In 27 patjents $(67,5 \%$ systodiastolic hypertension was found and in $12(30 \%$ only systolic hypertension was detected. Arterial hypertension persisted in 3 patients after the surgical treatment, in all of them a residual coarctation was demostrated.

Finally, we analize the principals mecanisms, which seem to play an important part in the production of hypertersion in Aortic Coarctation.
\end{abstract}

La Coartación Aórtica (Co Ao) es una anomalía congénita caracterizada por un estrechamiento o hipoplasia circunscrita de la aorta(Ao), ubicada próxima al ductus ${ }^{1}$. Morgagni la describió por primera vez en 1761 y Crafoord y Nylin realizaron en 1944 la primera intervención quirúrgica. Su incidencia seŕa de 1 por 10 a 12 mil pacientes pediátricos y se ha descrito asociada a otras malformaciones, tanto cardíacas como de otros sistemas, siendo más frecuente su presentación en el sexo masculino ${ }^{13}$.

Clínicamente se acompaña en más del $80 \%$ de los casos de hipertensión arterial (HA) en las extremidades superiores. Más frecuente aún sería la diferencia de intensidad de pulso entre las extremidades superiores e inferiores ${ }^{2}$. Según Londe el $2 \%$ de las HA de la infancia es consecuencia de $\mathrm{Co}$ Aol.

En los últimos años se han publicado varios estudios sobre la patogenia de la HA en estos pacientes, involucrándose factores mecáni$\cos ^{1-2-4-7}$, el sistema renina-angiotensina ${ }^{5-6-8 \cdot 9}$ a mecanismos similares a los del riffón de Goldblatt $^{10}$, no babiéndose demostrado todavía ninguno en forma fehaciente.

Según diferentes autores entre el 5 y $25 \%$ de los pacientes siguen hipertensos después de la intervención quirúrgica ${ }^{2-7-11}$, hecho que se ha relacionado con la edad de operación ${ }^{3-4}$, problemas de técnica quirúrgica ${ }^{2}$ y persistencia de los factores que condicionan la elevación de la presión en la $\mathrm{Co} \mathrm{Ao}^{2}$, siendo aún polémicas todas las explicaciones.

1 Médico becado de Pediatría, Hospital Luis Calvo Mackenna.

2 Médico Unidad de Nefrologia, Hospital Luis Calvo Mackenna.

${ }^{3}$ Médico Centro Cardiovasculat, Hospital Luis Calvo Mackenna.
Considerando estos antecedentes quisimos ana= lizar el comportamiento de la presión arterial (PA) en niños operados de Co Ao en el hospital Luis Calvo Mackenna y las probables causas de $\mathrm{HA}$ postoperatoria en nuestra serie.

\section{MATERLAL Y METODO:}

Se revisaron las estadísticas del Centro Cardiovascular del hospital Luis Calvo Mackenna entre los años 1975 y 1981, encontrándose 93 casos intervenidos por Co Ao. De ellos se analizaron 40 , pues el resto no cumplía con los requisitos de control postoperatorio por haber sido controlados en otros centros del país. Se descartaron además los casos en que se sospechó o comprobó una anomalía asociada cardíaca, renal o endocrina.

El análisis consideró los siguientes parámetros:

- Cifras de PA en el momento del diagnóstico.

- Edad de operación.

- PA postoperatoria.

El tiempo de control fluctuó entre los 3 meses y 5 años, más de los $2 / 3$ fueron controlados por 1 año 6 meses.

La PA se tomó en las extremidades superiores con esfingomanómetro de $\mathrm{Hg}$ y en las inferiores con oscilómetro, eligiendo el tercer valor para compararlo con las tablas de Nadas Y/o Norero y $\operatorname{cols}^{14}$.

La operación consistió en la resección de la zona estenótica y anastomosis término-terminal, En algunos casos, especialmente en los menores de un año de edad se practicó plastía aórtica con colgajo de subclavia.

\section{RESULTADOS:}

El análisis de las cifras de PA en la etapa preoperatoria mostró que: 
- De los 40 pacientes sólo uno era normotenso, significando un 97,5\% de pacientes hipertensos, cifra comparable con otras publicaciones ${ }^{3}$.

- La mitad de Jos pacientes consulta antes de Jos 4 affos de edad y el $32 \%$ antes del año de edad.

- Se eleva tanto la PA sistólica como diastólica siendo más significativa la elevación de la primera.

- 27 pacientes $(67,5 \%)$ presentaban $\mathrm{HA}$ sistólica y diastólica y $12(30 \%)$ sistólica exclusiva, dando el total de $97,5 \%$ de pacientes hipertensos. No encontramos pacientes que tuviesen sólo hipertensión diastólica.

El estudio postoperatorio de la PA se realizó en cada uno de los pacientes, encontrándose que:

- Tanto la PA sistólica como diastólica llega a valores normales, independiente de la edad de operación.

- EI descenso de la PA es independiente de la cifra de PA preoperatoria.

En las figuras 1 y 2 (página siguiente) se pueden apreciar las cifras de PA sistólica y diastólica de los 40 pacientes: en la etapa preoperatoria y como en el último control postoperatorio llegan a valores dentro del rango normal.

Làedad de operación fluctuó entre los 3 meses y 14 años. En la tabla 1 se observa que 9 pacientes

Tabla 1.

Coartación Aortica e Hipertensión Arterial (Edad de operación)

\begin{tabular}{lc}
\hline Edad & No de casos \\
\hline$<2$ años & 9 \\
$2-5$ & 7 \\
$>5$ & 24 \\
\hline TOTAL & 40 \\
\hline
\end{tabular}

(22\%) fueron intervenidos antes de los 2 años y 16 (40\%) antes de los 5 años de edad, cifra apropiada a la edad de diagnóstico y al criterio del Centro Cardiovascular que recomienda intervenir entre los 2 y 4 años de edad.

Tres pacientes $(7,5 \%)$ presentaban en el control postoperatorio hipertensión en las extremidades superióres con cifras menores y pulsos débiles en Jas inferiores, Tabla 2. En estos 3 pacientes se Tabla 2.

Coartación Aórtica e Hipertensión Arterial

(Pacientes hipertersos post operatorios)

Paciente P.A. pre op. Edad op. Evolución PA post op.

\begin{tabular}{lllll}
\hline I.S.. $\$ 10$ años & $140 / 80$ & 10 a $5 \mathrm{~m}$. & Sm. & $145 / 70$ \\
\hline E.R. $\$ 5 \mathrm{~m}$. & $150 / 90$ & 4 a $7 \mathrm{~m}$. & $1 \mathrm{a}$. & $140 / 85$ \\
\hline J.C. \$ 4 años & $140 / 80$ & 4 a $7 \mathrm{~m}$. & 2a. & $130 / 70$ \\
\hline
\end{tabular}

diagnosticó Co Ao residual atribuíble a problemas de técnico operatoria. Estos resultados concuerdan con información aportada por autores extranje$\operatorname{ros}^{2 \cdot 7 \cdot 11}$

Finalmente, en la Tabla 3 se pueden observar

Tabls 3.

Coestación Aórtica e Hipertedsión Arteria

Besión arterial pre y postoperatoris en $\mathrm{mm}$ de $\mathrm{Hg}(\mathrm{X} \pm \mathrm{DE})$

\begin{tabular}{|c|c|c|c|c|c|c|}
\hline \multirow{2}{*}{$\begin{array}{l}\text { EAlad } \\
\text { (Anos) }\end{array}$} & \multicolumn{3}{|c|}{ Preopetaloria } & \multicolumn{3}{|c|}{ Postoperaloria } \\
\hline & n & Sistoblica & Dfasıólica & $\pi$ & Sistólica & Diastóblica \\
\hline$<2$ & 16 & $162 \pm 24$ & $84 \pm 20$ & 4 & $108 \pm 11$ & $65 \pm 11$ \\
\hline$>2-5$ & 4 & $135 \pm 5$ & $71 \pm 7$ & 7 & $108 \pm 7$ & $62 \pm 6$ \\
\hline$>5$ & 20 & $148 \pm 21$ & $81 \pm 10$ & 29 & $112 \pm 12$ & $66 \pm 8$ \\
\hline
\end{tabular}

las cifras promedios $(\overline{\mathrm{X}})$ de presión arterial sistólica (PS) y diastólica (PD) y su Desviación Estandar, (DE), en los 40 pacientes, tanto en la etapa preoperatoria o primera consulta, como en la postoperatoria o última consulta. Los valores más elevados se obtuvieron en las PS de los menores de 2 aflos.

\section{DISCUSION:}

Se han postulado varios mecanismos paja explicar la HA en los pacientes con Co Ao. Uno de ellos es el factor mecánico obstructivo que al disminuir la cámara receptora del gasto cardíaco elevaría la PA sistólica. Otras publicaciones se refieren a la influencia del rifión, es así como se ha estudiado el sistema renina-angiotensina con resultados que parecen aún contradictorios $5-8-9-12$ En nuestra serie encontramos en $67,5 \%$ de los casos PA diastólica elevada, lo que podría deberse a la existencia de un factor vasocanstrictor como la angiotensina. Ribeiro ${ }^{5}$ demuestra que al usar Saralasina ${ }^{(\Theta)}$, un inhibidor de la angiotensina II, los pacientes con Co Ao disminuyen su PA, no asi los hipertensos esenciales. Alpert por otra parte post $\downarrow$ la un mecanismo similar al "Riñón de Goldblatt", secundario a la disminución del flujo renal y aumento de la secreción de renina. Sin embargo Amsterdam ${ }^{9}$ no encontró diferencias en la actividad de renina plasmática en niños con Co Ao y un grupo control normal.

Muchos trabajos se refjeren a la edad óptima para la intervención quirúrgica. Se señala que a los 4 años de edad el diámetro aórtico equivale al $50 \%$ del correspondiente en el adulto, permitiendo obviar el riesgo de HA residual ${ }^{2}$. Se recomienda operar entre los 2 y 5 antos $^{3-1} 1$ evitando asi las complicaciones de la $\mathbf{H A}$ como hemorragias intracraneanas, roturas de aneurismas, etc.

En cuanto a la HA postoperatoria, se han invocado factores como daño vascular ocurrido durante la etapa previa ${ }^{1}$, Co Ao residual, recoartación, existencia concomitante de estenosis de la arteria 
Figura 1.

Coartación Aóctica e Hipertensión Arterial (Presión arterial sistólica pre y post operatoria en 40 pacientes)

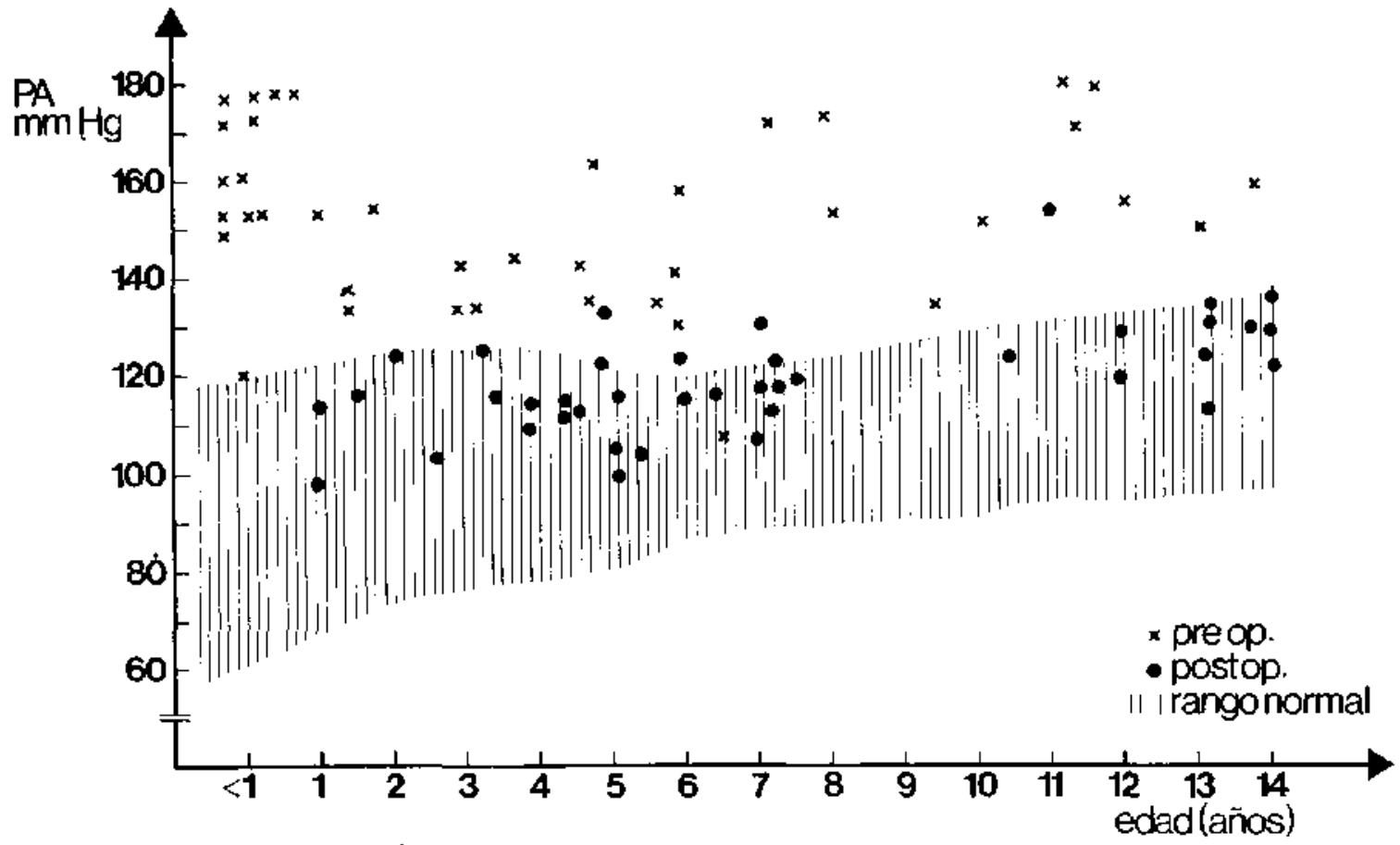

Figura 2.

Coartación Aórtica e Hipertensión Arterial (Presión arterial diastólica pre y post apcratoria en $\mathbf{4 0}$ pacientes)

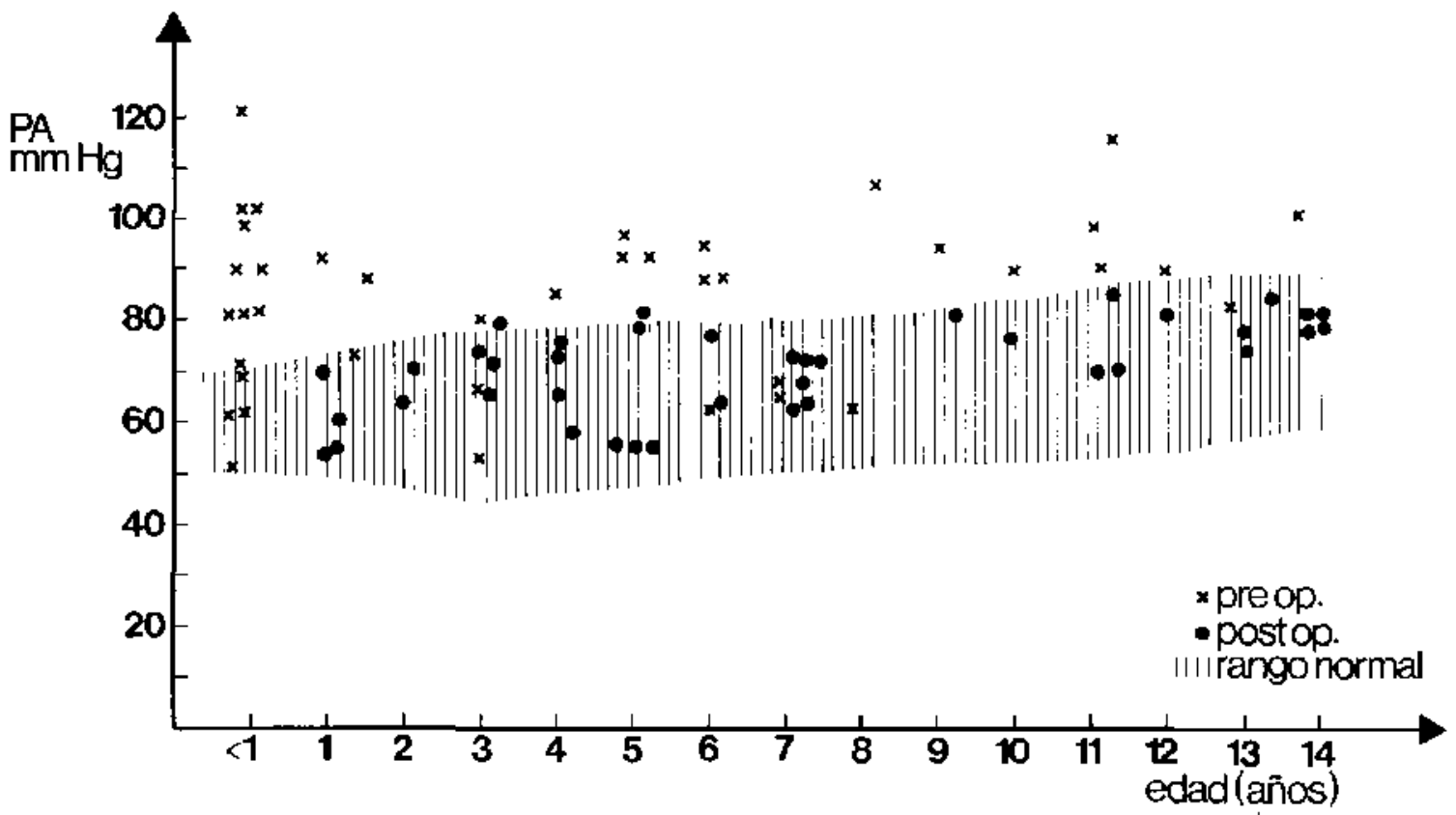


renal o HA esencial. En nuestra serie todas las $\mathrm{HA}$ postoperatorias se debieron a Co Ao residual y no hubo relación con la edad de operación.

\section{REFERENCIAS}

1 Londe, $S$. Causes of hypertension in the young. Ped. C. of N.A. 25: $\$ 53,1978$.

${ }^{2}$ Goldring, D. Treatment of the infant and child with Coarctation of the Aorta. Ped. Cl. of N.A. 25: 111, 1978.

${ }^{3}$ Liberthson, R., Peunington, D., Jacobs, M., Daggett, W. Coarctation of the Aorta. Review of 234 patients and clarification of management problems. Am. J. of Card. 43: 835, 1979.

4 Schested, J. Evaluation of optimun time for surgical repair of Coarctation of the Aorta. Surg. Gyn. Obst.: 146: $593,1978$.

5 Ribeiro, A., Krakoff, L. Angiotensin blockade in Coarctation of the Aorta. N. Eng. J. Med. 295: 148, 1976.

${ }^{6}$ Bagky, S., Wc Donald, W., Strong, D., et als.: Abnormalities of renal perfusion and the renal pressor system in dogs with chronic Coarctation of the Aorta. Circ. Res. 37: 615, 1975.
${ }^{7}$ Nanton, M. Olley, $P$. Residual hypertension after coarctectomy in children Am. J, of Card. 37: 769, 1976.

8 Morkiewicz, D., Wolczuk, D., Kokot, F., Cicha, A. Plasma renin activity in coarctation of the aorta before and after surgery. Br. Heart J. 37: 721, 1975.

${ }^{9}$ Amsterdom, E., Albers. W., Christlieb, R., et als. Plasma renin activity in children with coarctation of the aorta. Am. J. of Card. 23: 396, 1969.

10 Alpert, B., Bain, $H$., Williamson, $J$. et als. Role of the renin-angiotensinaldosterone system in hypestensive children with coarctation of the aorta. Am. J. of Card. 43: 828,1979 .

11 Nanton, $M$., Olley, P. Post op. blood pressure following coarctectomy in your children. Am. J. of Card. 33: 159, 1974.

12 Scott, W., Collins, H., Langa, A. et als. Additional observations concerning the physiology of the hypertension associated with experimental coarctation of the aorta. Surgery 36: 445, 1954.

13 Nelson, $W$. Textbook of Pediatrics. Cap. 13, pág. 1313. Ed. Saunder, 1979.

14 Nodos, A., Fyler. D. Cardiología pediátrica. Pág. 717. Ed. Interamericana, 1975. 